\title{
Retroperitoneal laparoscopic management of a solitary extramedullary plasmacytoma associated with human immunodeficiency virus infection: A case report
}

\author{
DEHONG CAO ${ }^{1 *}, \mathrm{YINGCHUN} \mathrm{HU}^{2 *}, \mathrm{LI} \mathrm{LI}^{3}$, WEI XIAO ${ }^{4}$ and QIANG WEI ${ }^{1}$ \\ Divisions of ${ }^{1}$ Urology and ${ }^{2}$ Geriatrics; ${ }^{3}$ Laboratory of Pathology, West China Hospital of Sichuan University, \\ Chengdu, Sichuan 610000; ${ }^{4}$ Division of Infectious Disease and Hospital Epidemiology, \\ Third Xiangya Hospital of Central South University, Changsha, Hunan 410013, P.R. China
}

Received November 3, 2014; Accepted August 20, 2015

DOI: $10.3892 / 01.2015 .3936$

\begin{abstract}
Extramedullary plasmacytoma (EMP) is a rare malignant tumor that is characterized by a malignant plasma cell neoplasm. Such neoplasms in human immunodeficiency virus (HIV)-infected patients are extremely rare. To the best of our knowledge, the present study describes the first case of a solitary adrenal EMP in a patient with HIV. A 35-year-old male who had been diagnosed with HIV 3 months previously presented with a 2-week history of intermittent right flank pain. Abdominal computed tomography revealed a soft-tissue density mass in the right adrenal gland area. The patient subsequently underwent a retroperitoneal laparoscopic adrenalectomy. Post-operative pathological diagnosis revealed a solitary EMP. Although the patient refused to undergo post-operative radiotherapy and chemotherapy, no recurrence was detected after 2 years of follow-up. The present case illustrates the fact that this rare type of solitary EMP associated with acquired immune deficiency syndrome (AIDS) can occur in the adrenal glands, and that retroperitoneal laparoscopic resection of the tumor may be a good method to manage this condition. In addition, although rare, solitary EMP should be considered in the differential diagnosis of an adrenal mass in HIV-infected patients.
\end{abstract}

Correspondence to: Professor Qiang Wei, Division of Urology, West China Hospital of Sichuan University, 37 Guoxue Alley, Chengdu, Sichuan 610000, P.R. China

E-mail: qiangwpro@163.com

*Contributed equally

Abbreviations: EMP, extramedullary plasmacytoma; HIV, human immunodeficiency virus; AIDS, acquired immune deficiency syndrome

Key words: extramedullary plasmacytoma, HIV, AIDS, adrenal, laparoscopic

\section{Introduction}

Extramedullary plasmacytoma (EMP) is a rare malignant tumor that is characterized by a malignant plasma cell neoplasm and accounts for $\sim 4 \%$ of all plasma cell malignancies (1). Additionally, $80 \%$ of such lesions are located in the head and neck region (2). A paucity of data exists concerning the presentation, natural course and outcome of EMP, however, Xing et al (3) reviewed 147 cases of EMP of the larynx. It was identified that most common treatment modality was radiotherapy alone. The mean survival duration was $\sim 184$ months, and the 5- and 10-year survival rates were $71.1 \%$ and $67.4 \%$, respectively. As the disease is a rare tumor, little information exists regarding the mortality rates. In addition, although certain patients have been treated with surgical excision, radiotherapy, chemotherapy, or combined surgery and radiotherapy, the exact treatment strategy and outcomes of EMP remain unclear. It also has no typical presentation or features, with diagnosis predominantly dependent on pathological analysis. In a literature search performed by a professional librarian using MEDLINE and EMBASE, it was found that studies on such neoplasms in HIV-infected patients are extremely rare, with only eight cases reported to date (4-10). To the best of our knowledge, the present study describes the first case of a solitary adrenal EMP in a patient with HIV.

\section{Case report}

A 35-year-old male who had been diagnosed with HIV 3 months previously presented to the West China Hospital of Sichuan University (Chengdu, China) in July 2013. The patient had a 2 -week history of intermittent right flank pain. The patient had a 2-year history of anal sexual intercourse. There was no family history or other medical history. Findings on physical examination were unremarkable. Abdominal computed tomography revealed a soft-tissue density shadow $\sim 35 \mathrm{~mm}$ in diameter, without enhancement, in the right adrenal gland area. Routine pre-operative examination showed no apparent abnormalities. Endocrine tests for adrenal hypersecretion were negative. The findings of routine blood tests, blood biochemical examination, routine urinalysis and renal function tests were also normal. 
A clinical diagnosis of a non-functioning adrenal tumor was suspected. Following communication with the patient and his family, a retroperitoneal laparoscopic adrenalectomy was performed. However, histopathological examination of the resected specimen suggested a plasmacytoma. Microscopy showed that the mass was composed of a dense and diffuse infiltrate of mature plasma cells with wheel spoke-like nuclei. A few mitotic figures were also apparent (Fig. 1). In addition, low-power microscopy revealed a small amount of reticular fibers running through the plasma cells (Fig. 2).

Subsequently, further post-operative investigations were performed. The serum immunoglobulin (Ig)G level was $18.7 \mathrm{~g} / \mathrm{l}$ (normal range, 8.0-15.5 g/l), however, the serum $\operatorname{IgA}$ and IgM levels were within the normal ranges. No Bence-Jones protein was detected in the urine. Immunohistochemical analysis showed positive cytoplasmic staining for $\lambda$-light chain. Subsequently, the bone marrow biopsy demonstrated $<3 \%$ plasma cells (diagnostic criteria, $<5 \%$ ). A full-body bone scan revealed no anomalies. Eventually, the patient was shown to meet all the required clinical and laboratory criteria for a solitary EMP. The patient recovered without complications and was discharged at 4 days post-surgery. Subsequently, the patient was administered allopathic anti-acquired immune deficiency syndrome (AIDS) drugs in the Sichuan Center for Disease Control and Prevention (Sichuan, China). No recurrence was detected after 2 years of follow-up.

\section{Discussion}

Plasmacytomas can be categorized as either EMP or medullary plasmacytoma (9). Each type is present in solitary and multiple forms. EMP is defined as an extraosseous proliferation of neoplastic plasma cells. The study by Juglard et al (11) suggested that the incidence of plasmacytoma in HIV-positive patients is greater than in non-infected patients. However, a solitary EMP associated with AIDS involving the adrenal gland is extremely uncommon. To the best of our knowledge, no case has been reported in the literature to date. Among the reported case reports involving EMP and infection by HIV, the locations of the tumors include the gingiva, the larynx, the central nervous system and the testes (4-10).

While the exact pathogenesis of EMP associated with HIV remains unclear, Herranz et al (12) suggested that the immune alterations, chronic viral infections and cytokine hyperexpression that exist in patients infected by HIV may act as the triggering stimulus for plasmacytoma to proliferate. The study also suggested that plasmacytoma may be another malignant hematological process associated with AIDS. In addition, a number of studies hypothesized that infectious agents, such as HIV, hepatitis $C$ virus and Epstein-Barr virus, may play a role in the pathogenesis of plasmacytoma (13-15). We agree with the current pathogenetic hypotheses, however, further studies are required for confirmation.

Although there are currently no clear treatment guidelines for solitary EMP associated with HIV, thus far, these patients have been treated with surgical excision, radiotherapy, chemotherapy, or combined surgery and radiotherapy. Ramadan et al (7) reported a patient with solitary testicular EMP combined with AIDS who was treated with combined surgical excision and radiotherapy. Hazarika et al (8)

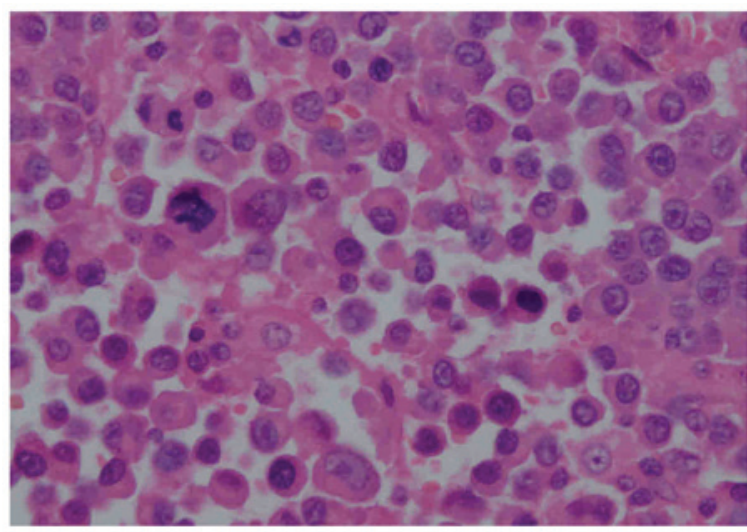

Figure 1. High-power microscopy showing the mass composed of a dense and diffuse infiltrate of mature plasma cells with wheel spoke-like nuclei. A few mitotic figures are also apparent (hematoxylin-eosin staining; magnification, $\mathrm{x} 400)$

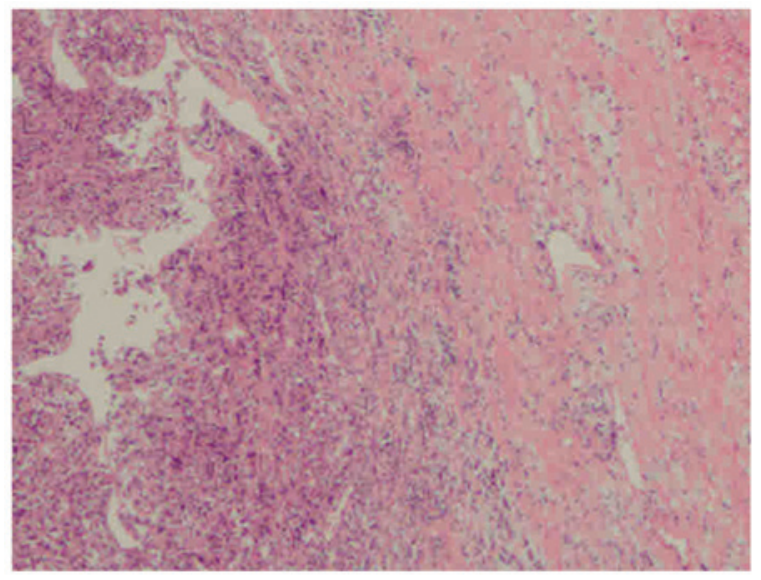

Figure 2. Low-power microscopy revealing a small amount of reticular fiber running through the plasma cells (hematoxylin-eosin staining; magnification, $\mathrm{x} 100$ )

reported a case of solitary sinonasal EMP with concomitant HIV, wherein the tumor mass was treated via radiotherapy. Wu et al (9) described the case of an HIV-positive individual with an solitary EMP of the central nervous system that was treated by chemotherapy. In addition, 2 other cases involving AIDS combined with EMP in the head and neck were reported by Bhattacharya et al (10), where the patients were treated with chemotherapy. In the present patient, retroperitoneal laparoscopic removal of the adrenal mass was successfully performed. Although the patient refused to undergo post-operative radiotherapy and chemotherapy, an uneventful recovery was experienced and the patient was discharged at 5 days post-surgery. The study suggest that the retroperitoneal laparoscopic resection may be a good method to manage this condition. Given the limited experience in treatment of EMP infection following HIV infection, further study is required to determine the appropriate regimen and duration of therapy.

Solitary EMP in HIV-positive patients tend to be more aggressive, with a poor prognosis. This may be due to the poor immunity of the patient. The study by Bhattacharya et al (10) identified that the mean survival time of two pa ti nets with 
HIV infection and EMP was $<6$ months after the original diagnosis was made. The present patient, however, was discharged after surgery and followed up for 2 years with no recurrence.

In conclusion, the present study illustrates the fact that this rare type of solitary EMP associated with AIDS can occur in the adrenal glands, and that retroperitoneal laparoscopic resection may be a good method to manage this tumor. In addition, although rare, solitary EMP should be considered in the differential diagnosis of an adrenal mass in HIV-infected patients.

\section{Acknowledgements}

The study was supported by the National Natural Science Foundation of China (grant nos. 81200551, 81270841 and 81300627).

\section{References}

1. Galieni P, Cavo M, Awisati G, Pulsoni A, Falbo R, Bonelli MA, Russo D, Petrucci MT, Bucalossi A and Tura S: Solitary plasmacytoma of bone and extramedullary plasmacytoma: Two different entities? Ann oncol 6: 687-691, 1995.

2. Husarić S, Pašić J, Alić E and Kuljanin M: Solitary extramedullary plasmacytoma of the liver. Acta Med Acad 42: 85-86, 2013.

3. Xing Y, Qiu J, Zhou ML, Zhou SH, Bao YY and Zheng ZJ: Prognostic factors of laryngeal solitary extramedullary plasmacytoma: A case report and review of literature. Int J Clin Pathol 8: 2415-2435, 2015.

4. Gastaut J, Quilichini R and Horchowski N: Localized extramedullary plasmacytomas (LEP) and HIV. In: IV International Conference on AIDS Program and Abstract Book. Vol. 2, p327, 1988.
5. Israel AM, Koziner B and Straus DJ: Plasmacytoma and the acquired immunodeficiency syndrome. Ann Intern Med 99: 635-636, 1983 .

6. Vallisa D, Pagani L, Bertè R, Civardi G, Viale P, Paties C and Cavanna L: Extramedullary plasmacytoma in a patient with AIDS: Report of a case and review of the literature. Tumori 84: 511-514, 1998.

7. Ramadan A, Naab T, Frederick W and Green W: Testicular plasmacytoma in a patient with the acquired immunodeficiency syndrome. Tumori 86: 480-482, 2000.

8. Hazarika P, Balakrishnan R, Singh R, Pujary K and Aziz B: Solitary extramedullary plasmacytoma of the sinonasal region. Indian J Otolaryngol Head Neck Surg 63: 33-35, 2011.

9. Wu W, Pasch W, Zhao X and Rezk SA: Extraosseous plasmacytoma with an aggressive course occurring solely in the CNS. Neuropathology 33: 320-323, 2013.

10. Bhattacharya AK, Han K and Baredes S: Extramedullary plasmacytoma of the head and neck associated with the human immunodeficiency virus. Ear Nose Throat J 77: 61-62, 1998.

11. Juglard R, Vidal V, Calvet P, Dussaut JP, Barea D, Colineau X, Tourrette JH and Solacroup JC: Plasmacytoma and AIDS: Unusual duodenal localization. J Radiol 82: 1729-1731, 2001 (In French).

12. Herranz S, Sala M, Cervantes M, Sasal M, Soler A and Segura F: Neoplasia of plasma cells with atypical presentation and infection by the human immunodeficiency virus. A presentation of two cases. Am J Hematol 65: 239-242, 2000.

13. Gold JE, Schwam L, Castella A, Pike SB, Opfell R and Zalusky R: Malignant plasma cell tumors in human immunodeficiency virus-infected patients. Cancer 66: 363-368, 1990.

14. Montella M, Crispo A, Russo F, Ronga D, Tridente V and Tamburini M: Hepatitis $C$ virus infection and new association with extrahepatic disease: Multiple myeloma. Haematologica 85: 883-884, 2000.

15. Sadeghian MH, Ayatollahi H, Keramati MR, Memar B, Jamedar SA, Avval MM, Sheikhi M and Shaghayegh G: The association of Epstein-Barr virus infection with multiple myeloma. Indian J Pathol Microbiol 54: 720-724, 2011. 